\title{
Review
}

\section{Perspective of the Human Body in Sasang Constitutional Medicine}

\author{
Junhee Lee, Yongjae Jung, Junghee Yoo, Euiju Lee and Byunghee Koh \\ Department of Sasang Constitutional Medicine, College of Korean Medicine, Kyunghee University, Seoul, \\ Republic of Korea
}

\begin{abstract}
The Sasang constitutional medicine (SCM), a medical tradition originating from Korea, is distinguished from the traditional Chinese medicine in its philosophical background, theoretical development and especially, the fundamental rationale that analyzes the structure and function of the human body within a quadrifocal scheme. In SCM, the structure of the body is comprehended within the Sasang quadrifocal scheme, and the function of the body is understood within the context of the energy-fluid metabolism and the water-food metabolism controlled by the four main organs (lung, spleen, liver and kidney). Also, the concept of Seong-Jeong is used to explain the structural and functional variations between different constitutional types that arise from the constitutional variations in organ system scheme, which are in turn caused by deviations in the constitutional Seong-Jeong. Therefore, understanding the SCM perspective of the human body is essential in order to fully appreciate the advantages of the constitutional typological system (which focuses on individual idiosyncrasies) found in SCM.
\end{abstract}

Keywords: Discourse on Organ Systems - Donguisusebowon - human body function - human body structure - Sasang constitution

\section{Introduction}

Lee Je-ma (1837-1900), who first discovered the Sasang constitutional medicine (SCM), defines in his work Donguisusebowon the four constitutional types (四象人, Sasangin): Taeyangin (太陽人, TY type), Soyangin (少陽人, SY type), Taeeumin (太陰人, TE type) and Soeumin (少陰人, SE type). He presented the physiology, pathology and guidelines for health specific to each constitutional type and described the constitutionally differentiated physical and psychological attributes. The SCM is a sub-division of the traditional Korean medicine (TKM) that studies the constitutional typology system based on these four constitutional types (1). SCM, an integral and prominent component of the TKM, is included in the official curriculum in the oriental medical

For reprints and all correspondence: Byunghee Koh, Department of Sasang Constitutional Medicine, Kyunghee Korean Medicine Hospital, 1 Hoegi-dong, Dongdaemun-gu, Seoul, 130-702, Republic of Korea.

Tel: +82-2-958-9231; Fax: + 82-2-958-9234;

E-mail: kmc2516@khmc.or.kr schools of Korea (2) and presently has wide clinical application in TKM practice. Also, 10-20 SCM specialists are being produced annually since 2002, when SCM was first accepted as one of the eight subdivisions of specialized TKM practice.

The concept of 'constitution' encompasses both the structural and functional aspects of the human body (3). Many studies have researched the constitutionally differentiated physical, mental, physiological and pathological qualities found in different constitutional types. The constitutionally differentiated attributes can be explained by the unique perspective of the human body seen in SCM. The SCM perspective of the human body is different from that of the conventional traditional Chinese medicine (TCM) in that it perceives the structural and functional system of the human body based on the quadrifocal scheme of the Sasang theory, as opposed to the Yin-Yang and Five Phases Theory that forms the basic rationale in TCM. This article was written in order to facilitate general understanding of SCM, an integral component of TKM, by introducing its

(C) 2009 The Author(s).

This is an Open Access article distributed under the terms of the Creative Commons Attribution Non-Commercial License (http://creativecommons.org/ licenses/by-nc/2.0/uk/) which permits unrestricted non-commercial use, distribution, and reproduction in any medium, provided the original work is properly cited. 
unique perspective of the human body based on the two chapters in Donguisusebowon, 'The Discourse on the Four Principles (四端論)' and 'The Discourse on Organ Systems (臟腑論)' $(4,5)$.

\section{The SCM Perspective of the Human Body Structure-A Structural Summary of the Human Body}

SCM examines the human body on multilevels along two axes: the superior-inferior dimension and the interiorexterior dimension. According to the chapter 'The Discourse on Organs (臟腑論)' of Donguisusebowon, the torso (including the head and the limbs in its division), is divided into four equal parts; the upper, the mid-upper, the mid-lower and the lower sector (also referred to as the four burn-centers).

The body consists of four Jang organs (四臟), namely the lung, spleen, liver and kidney, and four $B u$ organs (四腑), namely the esophagus, stomach, small intestine and large intestine, which are in turn controlled by the four Jang organs. The lung, spleen, liver and kidney in SCM are not simply derived from the Five Viscera in TCM (minus the heart); in SCM, the body is compartmentalized into four vertical sections which are each represented by the lung, spleen, liver and kidney. Although the four Jang organs in SCM share certain similarities in their functions with the Five Viscera in
TCM, the concept of lung, spleen, liver and kidney possesses both structural and functional qualities. Likewise, the four $B u$ organs (namely the esophagus, stomach, small intestine and large intestine) in SCM are different from the six bowels (namely the gall bladder, small intestine, stomach, large intestine, urinary bladder and triple burner) in TCM; in SCM, the gastrointestinal tract is segmented into four parts corresponding to the four burn-centers, resulting in the four $B u$ organs (which includes the esophagus, formerly not seen included in the six bowels).

Furthermore, SCM assigns certain body parts to associated Jang organs (lung, spleen, liver and kidney) and the $B u$ organs (esophagus, stomach, small intestine and large intestine). The body parts related to the lung, spleen, liver and kidney are compartmentalized in the posterior part of the body, whereas those related to the esophagus, stomach, small intestine and large intestine are compartmentalized in the anterior part of the body. The lung, spleen, liver and kidney are each associated with the nape area, the upper back area, the lower back area and the gluteal area, respectively; the esophagus, stomach, small intestine and large intestine are each associated with the supra-thoracic area, the thoracic area, the umbilical area and the infra-umbilical area, respectively (Fig. 1).

Other structures are also assigned accordingly-the skin, muscle, flesh and bone tissues are assigned to the

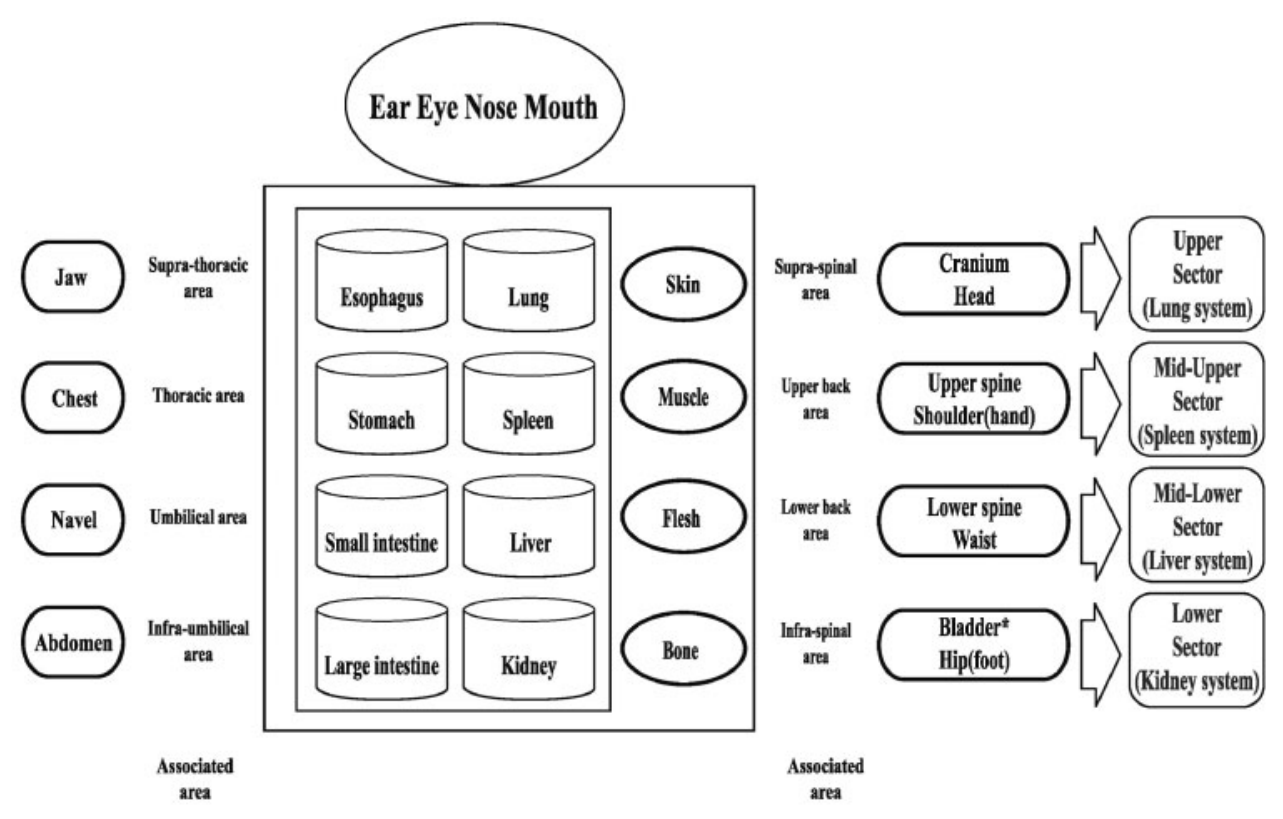

* The bladder in SCM refers to the region below lower back area

Figure 1. The structural scheme of the human body in SCM. The body consists of four Jang organs (四臟), namely the lung, spleen, liver and kidney, and four $B u$ organs (四腑), namely the esophagus, stomach, small intestine and large intestine, which are in turn controlled by the four $J a n g$ organs, and specific body parts are associated with the Jang organs and the Bu organs. Organs and related body components constitute the four systems (四黨). 
upper, mid-upper, mid-lower and lower burn-centers, respectively. Although the face is not mentioned in particular, the facial sensory organs (ear, eye, nose and mouth) are featured importantly in the original manuscripts, extending beyond the role as simple sensory receptors to encompass the functions of perception, judgment and cogitation. It can therefore be understood that certain cerebral functions are represented by these external sensory organs.

In conventional TCM, the heart (or Shim) is indeed described as 'the sovereign organ (君主之官)' that has a double role, the structural role as the 'heart (心藏)' within the circulatory system and the functional role as the 'mind (心)' that controls the mental and physical conditions of the human body. And yet, despite this particularity, the heart is still placed within the five phases to represent the Fire Elemental-Phase [and therefore subject to the generating (相生) and restraining (相克) laws of the Five Phases]. In SCM, the human body is compartmentalized into the mental component (the mind) and the physical component (the body), whereby the body consists of the lung, spleen, liver and kidney, and the mind is represented by the heart that acts as the structural and functional controller of the entire human body superior to the other organs (1). In the chapter 'The Discourse on Organ Systems (臟腑論)' of Donguisusebowon, the heart is described as 'the supreme ruler of the whole body'.

\section{The SCM Perspective of the Human Body Function-A Functional Summary of the Human Body}

\section{The Key Functional Elements}

In SCM, the physiological functions of the body are mainly controlled by the lung, spleen, liver and kidney. The metabolic activity that takes place within the human body largely falls into one of the two categories: the water-food metabolism (水穀代謝) and the energy-fluid metabolism (氣液代謝). The water-food metabolism pertains to the nutritional metabolism of the body that includes the ingestion and digestion of food, absorption and distribution of nutrients, actual processing within tissues and the generation and excretion of the resultant metabolic waste material-hence, water-food (水嗀) refers to the absorption, metabolizing and excretion processes involving the gastrointestinal tract. The energyfluid metabolism pertains to the energy metabolism of the body that includes the inter-conversion between energy and substance and the generation and consumption of energy - hence, energy-fluid (氣液) refers to the energy and bodily fluids that provide basis for the comprehensive human body functions.

The water-food metabolism is controlled by the spleen and the kidney. The spleen is involved in containing and accumulating the nutrient material while the kidney is involved in discharging and expelling the nutrient material. Therefore, the spleen and kidney can be described as the storeroom that controls the drawing in and sending out of nutrient material. The energy-fluid metabolism is controlled by the lung and the liver. The lung is involved in exhaling and dispersing the energy and fluid while the liver is involved in inhaling and concentrating the energy and fluid. Therefore, the lung and liver can be described as the gateway that controls the exhaling and inhaling of energy and fluid.

The four $B u$ organs (esophagus, stomach, small intestine and large intestine) are controlled by the four Jang organs (lung, spleen, liver and kidney). In other words, the containing and accumulating of the nutrient material in the stomach is controlled by the spleen, the discharging and expelling of the nutrient material in the large intestine is controlled by the kidney, the exhaling and dispersing of the energy and fluid in the esophagus is controlled by the lung and the inhaling and concentrating of the energy and fluid in the small intestine is controlled by the liver (Fig. 2).

In 'The Discourse on the Four Principles (四端論)' of Donguisusebowon, the functional attributes of the lung energy, spleen energy, liver energy and kidney energy are described as follows: the lung energy straightens and spreads, the spleen energy shells and embraces, the liver energy tolerates and loosens, and the kidney energy stacks and accumulates. In 'The Discourse on Organ Systems (藏腑論)' of Donguisusebowon, the functions of the esophagus, stomach, small intestine and large intestine are each evaluated based on anatomical study and its functional role in processing the foodstuff, which are described as follows: the esophagus contains ascending energy, the stomach contains containing energy, the small intestine contains consuming energy and the large intestine contains descending energy.

\section{The Basic Unit of Functional Performance and Structural Formation}

\section{The Generation of the Basic Units-The Generation of the Four Energies (四氣)}

The basic units that form the body structure and perform its functions are derived from the four energies (warm energy, hot energy, cool energy and cold energy) extracted from foodstuff. The four energies are created as the ingested foodstuff passes down the four $B u$ organs in the gastrointestinal tract. The process of energy formation is as follows: first, the foodstuff enters through the mouth into the stomach, where it is contained and steamed to generate hot energy. The light and clear component of the hot energy ascends to the esophagus and converts into warm energy. The foodstuff continues to move from the stomach to the small intestine where it 


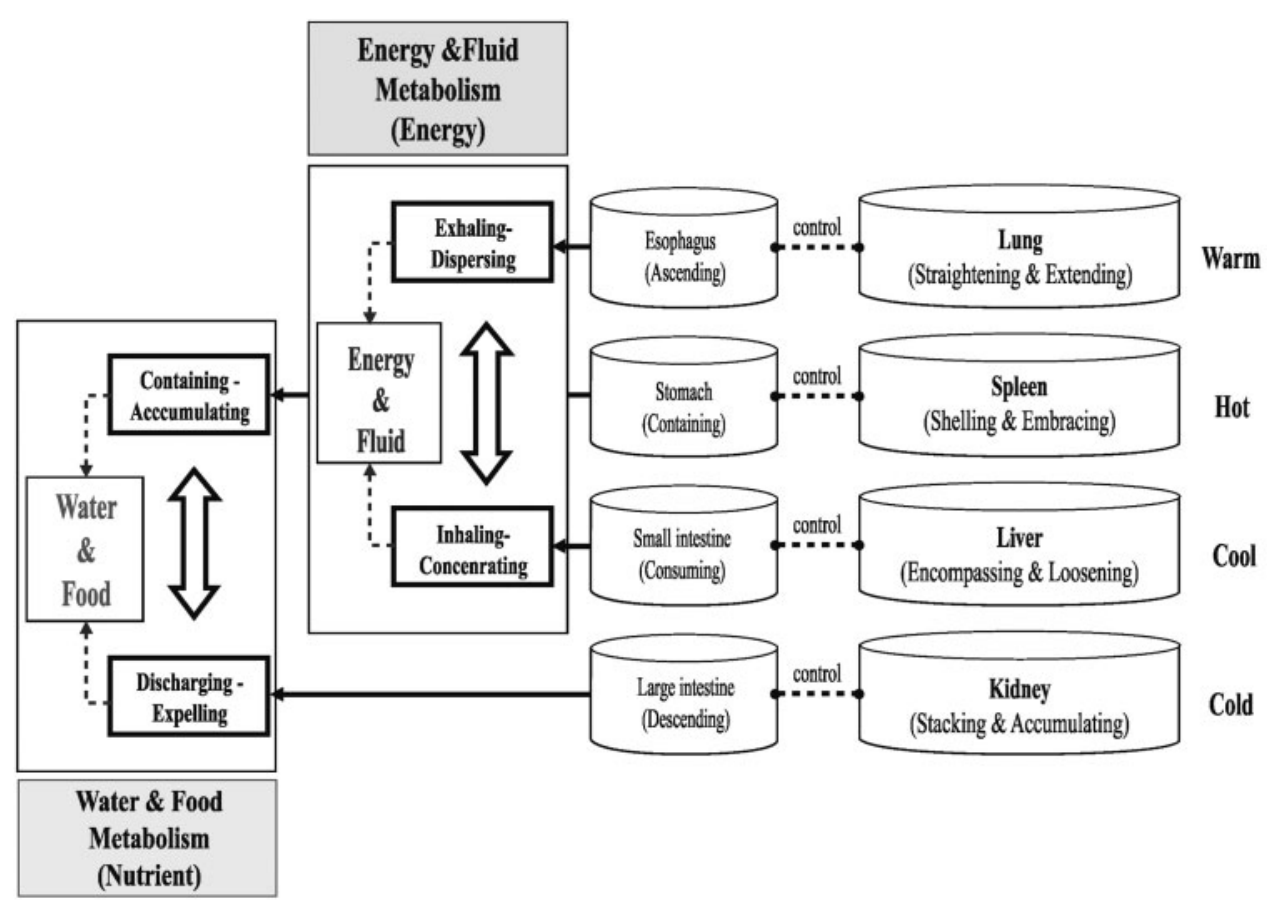

Figure 2. The functional scheme of the human body in SCM. In SCM, the physiological functions of the body are mainly controlled by the lung, spleen, liver and kidney. The metabolism taking place within the body is largely divided into the energy-fluid metabolism and the water-food metabolism: the energy-fluid metabolism is controlled by the liver and the lung, whereas the water-food metabolism is controlled by the spleen and the kidney.

is consumed and distilled to generate cool energy. The heavy and thick component of the cool energy descends to the large intestine and converts into cold energy. The hot energy and the cool energy are primary energies derived directly from the foodstuff, whereas the warm energy and the cold energy are secondary energies resulting from energy conversion. The hot energy and the cold energy are the basic units in the water-food metabolism, whereas the warm energy and the cool energy are those in the energy-fluid metabolism.

\section{The Circulation of the Four Energies (四氣)}

The four energies (warm energy, hot energy, cool energy and cold energy) each circulate their corresponding burncenters, through which process the structural and functional basic units are formed. The basic structural units include the Jin-Go-Yu-Aek (津 亳 油 液) material and the Ni-Mak-Hyeol-Jeong (淢 膜 血 精) material; the basic functional units include the Shin-Gi-Hyeol-Jeong (神氣 血 精) material. These basic units all carry the qualities of the corresponding type of energy among the four energies, the warm energy, hot energy, cool energy and cold energy. The Jin-Go-Yu-Aek material accumulates in the anterior parts of the four burn-centers that are referred to as the four anterior pools. The Jin-Go-YuAek material, the basic substance of the four anterior pools, is classified according to the site of accumulation and its characteristics: the $\operatorname{Jin}$ (津) material is the thin fluid accumulating in the upper anterior pool, the Go (亳) material is the pasty fluid accumulating in the mid-upper anterior pool, the $Y u$ (油) material is the oily fluid accumulating in the mid-lower anterior pool and the Aek (液) material is the thick fluid accumulating in the lower anterior pool. The Ni-Mak-Hyeol-Jeong material accumulates in the posterior part of the four burn-centers that are referred to as the four posterior pools. The Ni-Mak-Hyeol-Jeong material, the basic substance of the four posterior pools, is classified according to the site of accumulation and its characteristics: the $N i$ (淢) material is the greasy fluid accumulating in the upper posterior pool, the Mak (膜) material is the membranous fluid accumulating in the mid-upper posterior pool, the Hyeol (血) material is the blood-like fluid accumulating in the mid-lower posterior pool, and the Jeong (精) material is the essencelike fluid accumulating in the lower posterior pool.

The four energies (warm energy, hot energy, cool energy and cold energy) generated in the esophagus, stomach, small intestine and large intestine are first converted into the Jin-Go-Yu-Aek material and each enter the sublingua, intermammilla, navel and groin to form the four anterior pools (pool of Jin, pool of Go, pool of $Y u$ and pool of Aek). The Jin-Go-Yu-Aek material thus stored in the four anterior pools separate into clear energies (淸氣) and thick dregs (濁滓) that each travel different pathways. The clear energies each fill the corresponding burn-centers and 


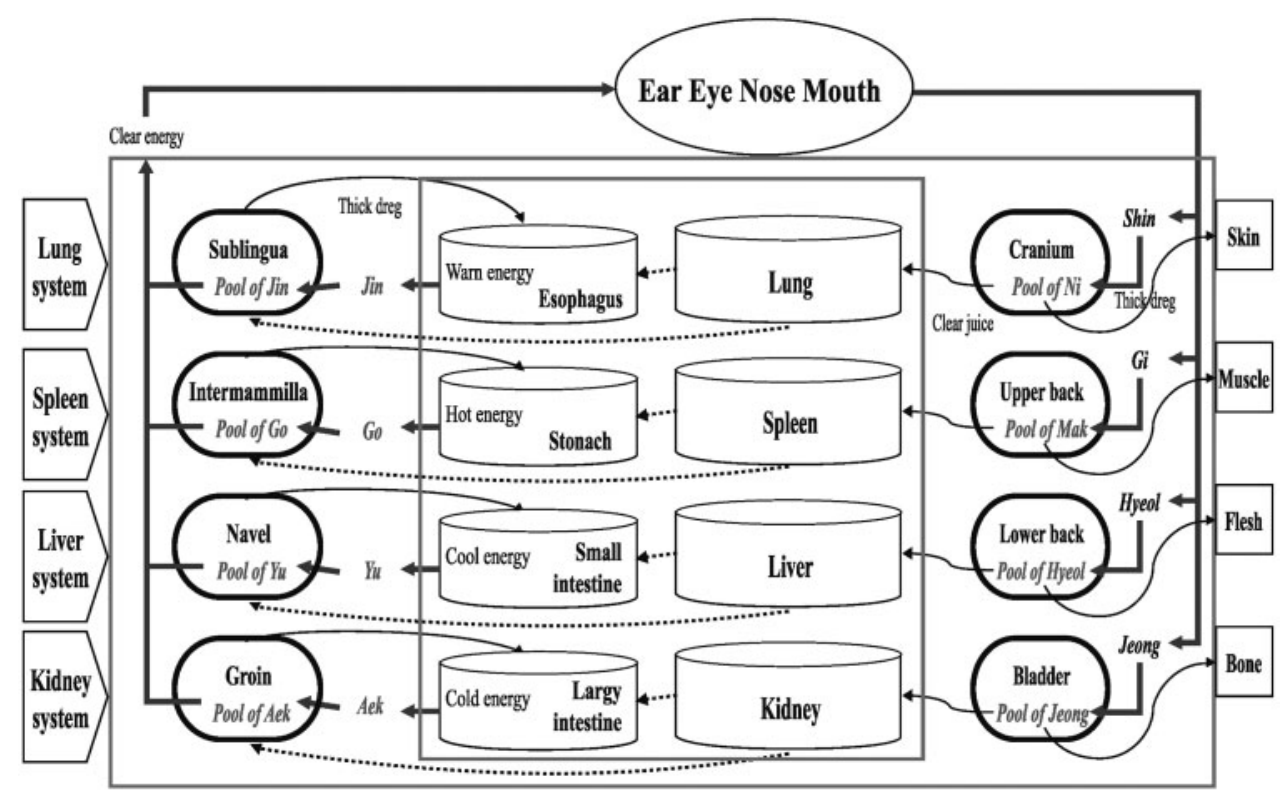

Figure 3. The circulation of the four energies. The four energies (warm energy, hot energy, cool energy and cold energy) each circulate their corresponding burn-centers, through which process the structural and functional basic units are formed. Jin (津), thin fluid; Go (言), pasty fluid; $Y u$ (油), oily fluid; Aek (液), thick fluid; $N i$ (䧕), greasy fluid; Mak (膜), membranous fluid; Hyeol (血), blood-like fluid; Jeong (精), essence-like fluid; Shin (神), spirit; Gi (氣), energy; Hyeol (血), blood; Jeong (精), essence.

convert into the basic functional units of Shin-Gi-HyeolJeong, which carry out the physiological functions of the body. The thick dregs convert into the basic structural units that activate the functions of the esophagus, stomach, small intestine and large intestine.

The Shin-Gi-Hyeol-Jeong material which constitute the functional basic units of the four burn-centers each enter the cranium, upper spine, lower spine and urinary bladder and convert into Ni-Mak-Hyeol-Jeong material before congealing to form the four posterior pools (pool of $\mathrm{Ni}$, pool of Mak, pool of Hyeol and pool of Jeong). The Ni-Mak-Hyeol-Jeong material thus stored in the four posterior pools separate into clear juices (清汁) and thick dregs (濁滓) that each travel different pathways. The clear juices activate the functions of the lung, spleen, liver and kidney and catalyze the formation of the four anterior pools. The thick dregs provide the material basis for the skin, muscle, flesh and bone.

To summarize, Jin-Go-Yu-Aek material in the four anterior pools and Ni-Mak-Hyeol-Jeong material in the four posterior pools act as basic structural units that form the body structure and provide the material base necessary to activate the functions of the organs (the lung, spleen, liver and kidney; the esophagus, stomach, small intestine and large intestine), the main components of the human body. The Shin-Gi-Hyeol-Jeong material act as basic functional units for the functions of the four burn-centers. In other words, the four energies (warm energy, hot energy, cool energy and cold energy) convert into Shin-Gi-Hyeol-Jeong, Jin-Go-Yu-Aek and Ni-Mak-Hyeol-Jeong materials which are used to form body structures and perform bodily functions.

In SCM, organs and related body components are classified into four systems (四黨) derived from the metabolic pathway of the four energies. The lung system comprises the esophagus, sublingua, ear, cranium and skin; the spleen system comprises the stomach, intermammilla, eye, upper back and muscle; the liver system comprises the small intestine, navel, nose, lower back and flesh; and the kidney system comprises the large intestine, groin, mouth, urinary bladder and bone. Each of the four systems contains one of the four energies and provides the metabolic pathway through which the four energies (warm energy, hot energy, cool energy and cold energy) are converted to form structures and carry out functions (Fig. 3).

\section{The Driving Forces in the Four Energy Circulation}

The aforementioned circulation of the four energies and the metabolic pathway of energy conversion are maintained by the four driving forces: force driven by the ear, eye, nose and mouth; force driven by the lung, spleen, liver and kidney; force driven by the esophagus, stomach, small intestine and large intestine; and force driven by the head, hand, waist and foot.

(i) By the force controlled by the ear, eye, nose and mouth, the clear energies of the four anterior pools are drawn to fill the upper, mid-upper, mid-lower and lower burn-centers controlled by the lung, spleen, liver and kidney, and then converted into 


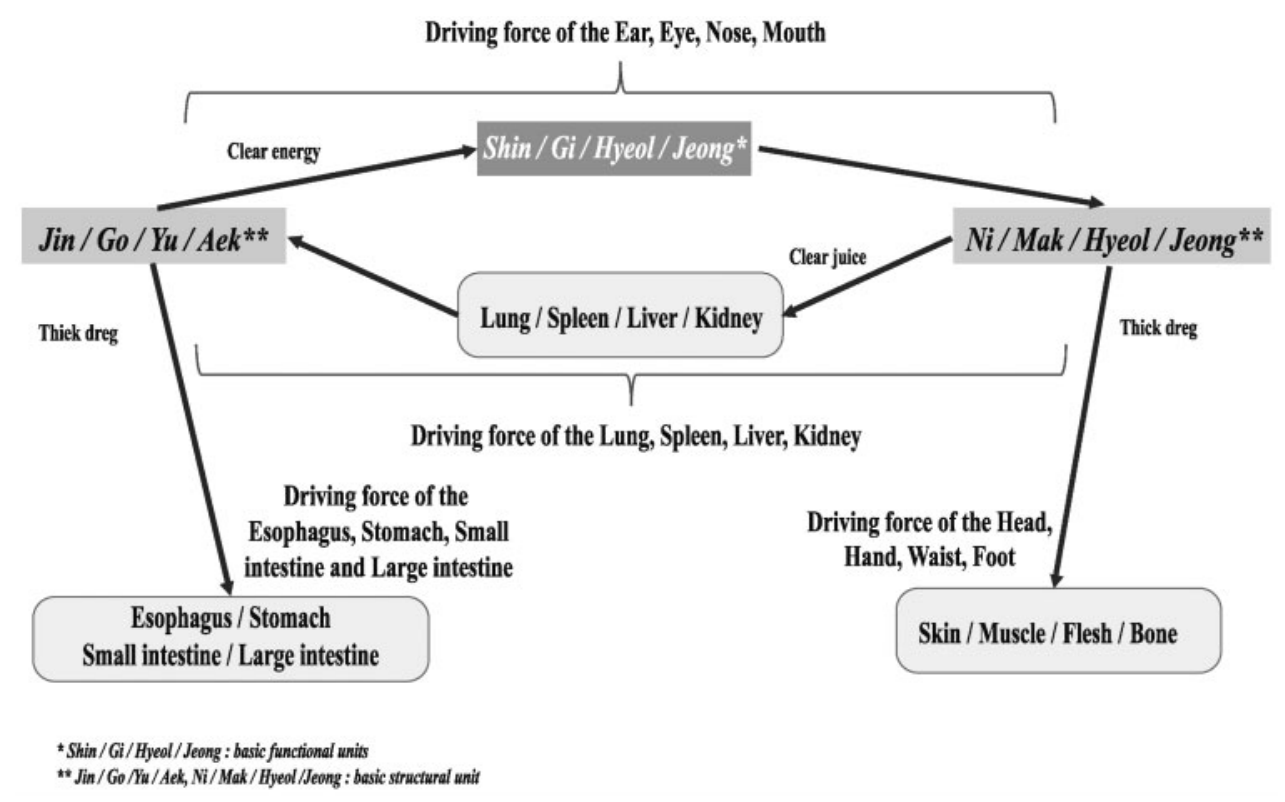

Figure 4. The driving forces in the four energy circulation. The circulation of the four energies and the metabolic pathway of energy conversion are maintained by the four driving forces.

Shin-Gi-Hyeol-Jeong material to perform bodily functions. The Shin-Gi-Hyeol-Jeong material are drawn into the cranium, upper back, lower back and urinary bladder to form the four posterior pools (pool of $\mathrm{Ni}$, pool of Mak, pool of Hyeol and pool of Jeong).

(ii) By the force controlled by the lung, spleen, liver and kidney, the clear juices of the four posterior pools are drawn into the lung, spleen, liver and kidney to nourish the lung, spleen, liver and kidney source, and then used to activate the four anterior pools (pool of Jin, pool of Go, pool of $Y u$ and pool of $A e k$ ) to facilitate the formation of the Jin-Go-Yu-Aek material.

(iii) By the force controlled by the esophagus, stomach, small intestine and large intestine, the ascending, containing, consuming and descending energies use the thick dregs of the four anterior pools to strengthen and fortify the esophagus, stomach, small intestine and large intestine.

(iv) By the force controlled by the head, hand, waist and foot, the extending, grasping, releasing and flexing energies use the thick dregs of the four posterior pools to form the skin, muscle, flesh and bone.

The four $B u$ organs form the basic structural units of the four anterior pools (pool of Jin, pool of Go, pool of $Y u$ and pool of Aek), which is in turn controlled by the driving force of the ear, eye, nose and mouth, and manifested as the bodily functions of Shin-Gi-HyeolJeong and eventually converted into the basic structural units of the four posterior pools (pool of $\mathrm{Ni}$, pool of
Mak, pool of Hyeol and pool of Jeong). The four Jang organs use the structural unit of the four posterior pools to activate themselves and simultaneously assist the four $B u$ organs and contribute to the formation of the four anterior pools (pool of Jin, pool of Go, pool of $Y u$ and pool of Aek).

The circulation and conversion of the four energies described above can be seen as a feedback process that helps maintain homeostasis and is therefore an expression of the ability to maintain structural and functional balance through constant interaction. Especially, the main controlling component of this process (the ear, eye, nose and mouth, and the lung, spleen, liver and kidney) holds supremacy over the overall process and, though not specifically mentioned, can be said to have similar functions, as that of the brain in modern medicine (6) (Fig. 4).

\section{The Formation of the Four Constitutional Types - The Structural and Functional Variations and Deviations}

\section{The Human Being in the SCM Perspective}

The SCM perspective of the human body is based on its perspective of the human being. The human being in the SCM perspective is explained within the context of the correlation between the human being (as the subject) and the non-human elements (as the object). In other words, the life of the human being is a series of the relationship he has with the society, environment and other human beings. In SCM, the objective elements, 
as opposed to the subjective human being, are explained with two concepts that have different qualities: the heavenly loom (天機) and the humanly affair (入事). Certain anatomical body parts and expressions were used to symbolize the subjective human action (e.g. ear, eye, nose, mouth and lung, spleen, liver, kidney). The act of observing the heavenly loom (one of the objective elements) by the human being (the subjective body) is expressed as the ear, eye, nose, mouth observing (hearing, seeing, smelling, tasting) the heavenly loom, and the act of performing the humanly affair (one of the objective elements) by the human being (the subjective body) is expressed as the lung, spleen, liver, kidney performing the humanly affair.

The aggregate of the cognitive and judgmental qualities formed during the subjective observation of the objective bodies (as the ear, eye, nose and mouth observes the heavenly loom) is called the Seong (性), and the aggregate of the responsive and behavioral qualities formed during the interaction of the objective and subjective bodies (as the lung, spleen, liver and kidney performs the humanly affairs) is called the Jeong (情). Henceforth, the Seong-Jeong (性情) is defined within the relationship between the subjective and objective elements. The Seong-Jeong (性情) was originally used in Confucianism to define the human nature, and in conventional
Confucian schools, the Seong is the universal law and innate nature and Jeong the emotional manifestation generated when the mind responds to the external elements. However, in SCM, the concept of Seong-Jeong (性情) encompasses all the qualities formed during the cognition, judgment, reaction and action that takes place in the interaction between the object and the subject. It has a predetermined yet individually differentiated quality. The Seong and Jeong in SCM are expressed as 'sorrow', 'anger', 'joy' or 'pleasure', and each of the four constitutional types has a specific predetermined pattern of Seong and Jeong. The terms 'joy', 'anger', 'sorrow' and 'pleasure' express more than simple humanly emotionsthey implicate qualities related to cognition, cogitation, judgment, reaction and action. For example, the TY type is characterized by the Seong of 'sorrow' and Jeong of 'anger' and henceforth expresses the Seong of sorrow and Jeong of anger.

The SCM perspective of the human body is based on its perspective of the human being explained above, and likewise it is understood that the 'smallness' and 'largeness' of the organ systems are formed based on the constitutionally differentiated patterns of Seong-Jeong deviation. The internal Seong-Jeong deviation and organ system scheme are externally manifested as the physical, mental and physiopathological traits (Fig. 5).

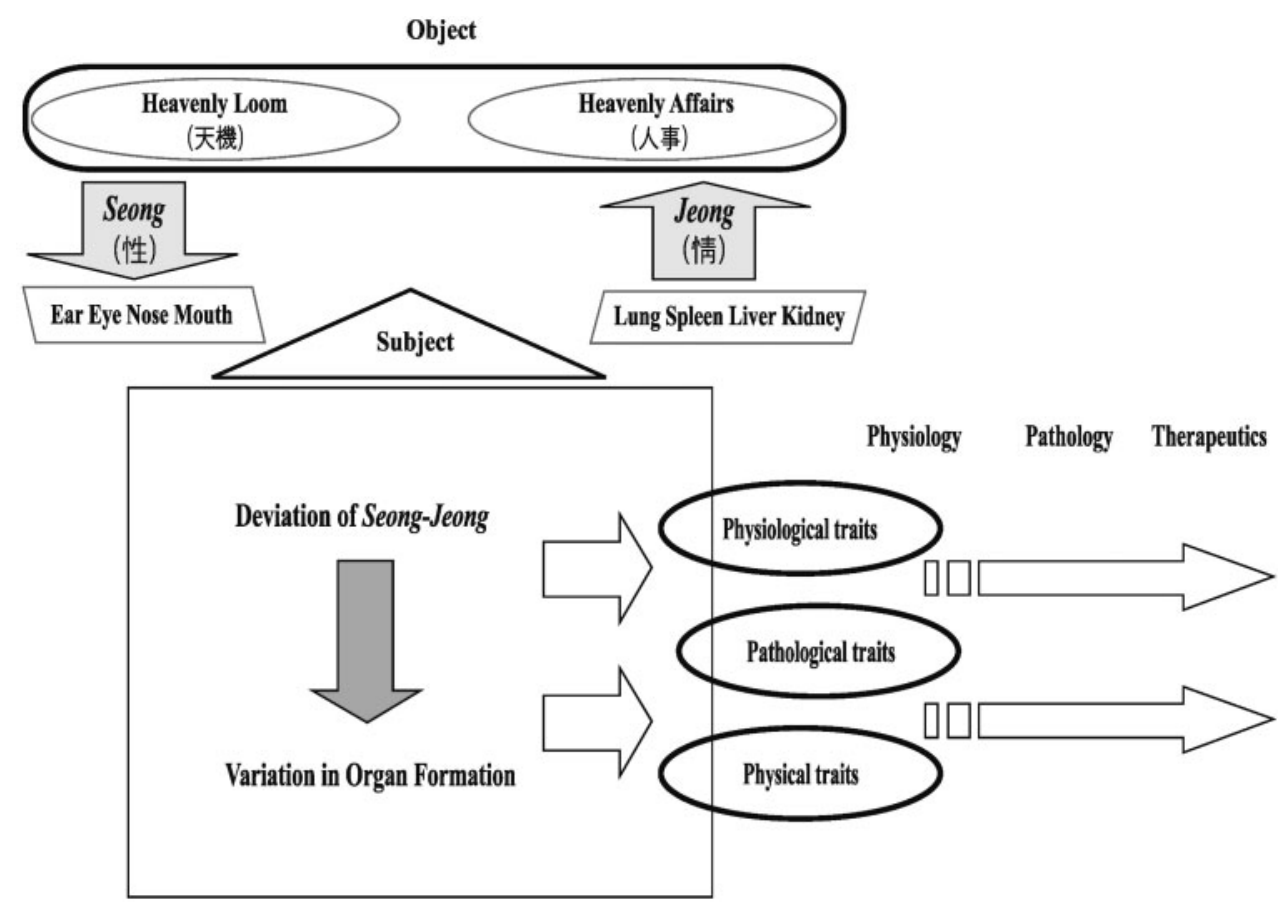

Figure 5. The human being in the SCM perspective. The aggregate of the cognitive and judgmental qualities formed during the subjective observation of the objective bodies (as the ear, eye, nose and mouth observes the heavenly loom) is called the Seong (性), and the aggregate of the responsive and behavioral qualities formed during the interaction between the objective and subjective bodies (as the lung, spleen, liver and kidney performs the humanly affairs) is called the Jeong (情). The internal Seong-Jeong deviation and organ system scheme are externally manifested as physical, mental and physiopathological traits. 


\section{The Energy of 'Sorrow (哀)', 'Anger (怒)', 'Joy (喜)' and 'Pleasure (樂)'}

Seong-Jeong within the human being has the quality of sorrow, anger, joy and pleasure, and although these terms are generally used to express emotional states, the 'sorrow', 'anger', 'joy' and 'pleasure' in SCM are perceived to be energies that can exert influence on the flow of energy within the human body. The Seong-Jeong incurs the following changes in energy directionality within the body through 'sorrow', 'anger', 'joy' and 'pleasure'. Sorrow causes energy to move straight upward, anger causes energy to move obliquely upward, joy causes energy to move obliquely downward and pleasure causes energy to move straight downward. The changes in energy caused by 'sorrow', 'anger', 'joy' and 'pleasure' influence the formation of organ systems. The energy moving straight upward due to sorrow enters and accumulates in the lung system, the energy moving obliquely upward due to anger enters and accumulates in the spleen system, the energy moving obliquely downward due to joy enters and accumulates in the liver system and the energy moving straight downward due to pleasure enters and accumulates in the kidney system.

\section{Organ Formation by the Seong-Jeong of Sorrow, Anger, Joy and Pleasure}

The Seong of sorrow, anger, joy and pleasure causes energy to move evenly, thereby adding to the associated organ system and making it 'larger'. To propound, the Seong of sorrow makes the lung system 'larger', the Seong of anger makes the spleen system 'larger', the Seong of joy makes the liver system 'larger' and the Seong of pleasure makes the kidney system 'larger'.

The Jeong of sorrow, anger, joy and pleasure causes energy to move unevenly and acutely, thereby detracting from the organ system associated with the opposite energy and making it 'smaller'. To propound, the Jeong of sorrow causes energy to move straight upward in uneven and acute manner, which undermines the movement of energy caused by pleasure (straight downward movement); the Jeong of pleasure causes energy to move straight downward in uneven and acute manner, which undermines the movement of energy caused by sorrow (straight upward movement); the Jeong of anger causes energy to move obliquely upward in uneven and acute manner, which undermines the movement of energy caused by joy (obliquely downward movement) and the Jeong of joy causes energy to move obliquely downward in uneven and acute manner, which undermines the movement of energy caused by anger (obliquely upward movement). As result, the organ systems associated with the undermined energy of sorrow, anger, joy and pleasure are damaged and eventually become 'smaller'; the Jeong of sorrow makes the kidney system 'smaller', the Jeong of anger makes the liver system 'smaller', the Jeong of joy makes the spleen system 'smaller', and the Jeong of pleasure makes the lung system 'smaller'.

\section{The Formation of 'Small' and 'Large' Organ Systems and the Resultant Structural and Functional Deviations}

Each constitutional type has a specific pattern of SeongJeong deviation, and this deviation causes the variations in the formation of organ systems (with specific patterns of 'smallness' and 'largeness') in different constitutional types. The organ formation in different constitutional types is as follows: the Seong-Jeong pattern of Seong of sorrow and Jeong of anger is characteristic of the TY type, leading to the formation of its specific organ scheme with a 'large' lung system and a 'small' liver system; the Seong-Jeong pattern of Seong of anger and Jeong of sorrow is characteristic of the SY type, leading to the formation of its specific organ scheme with a 'large' spleen system and a 'small' kidney system; the Seong-Jeong pattern of Seong of joy and Jeong of pleasure is characteristic of the TE type, leading to the formation of its specific organ scheme with a 'large' liver system and a 'small' lung system; and the Seong-Jeong pattern of Seong of pleasure and Jeong of joy is characteristic of the SE type, leading to the formation of its specific organ scheme with a 'large' kidney system and a 'small' spleen system. The constitutionally differentiated organ system scheme (with its pattern of 'large' and 'small' organ systems) thus formed from the SeongJeong deviation gives rise to the constitutionally differentiated physiological traits. This physiological variation can be explained by the SCM perspective on the structure and function of the human body.

The Taeyangin and Taeeumin share a physiological system based on the relationship and balance between the exhalant and dispersive function of the lung system and the inhalant and concentrative function of the liver system, two main components of the energy-fluid metabolism. Due to the organ system scheme of a 'large' lung system and a 'small' liver system in Taeyangin, the structure and function of body components associated with the lung system are relatively developed, whereas those associated with the liver system are relatively undeveloped. The exterior physical elements associated with the lung system are relatively developed, whereas those associated with the liver system are relatively undeveloped. The exhaling and dispersing of energy and fluid carried out by the lung and esophagus are relatively strong, whereas the inhaling and concentrating of energy and fluid carried out by the liver and small intestine are relatively weak. Due to the organ system scheme of a 'large' liver system and a 'small' lung system in Taeeumin, the structure and function of body components associated with the liver system are relatively developed, whereas those associated with the lung system are 


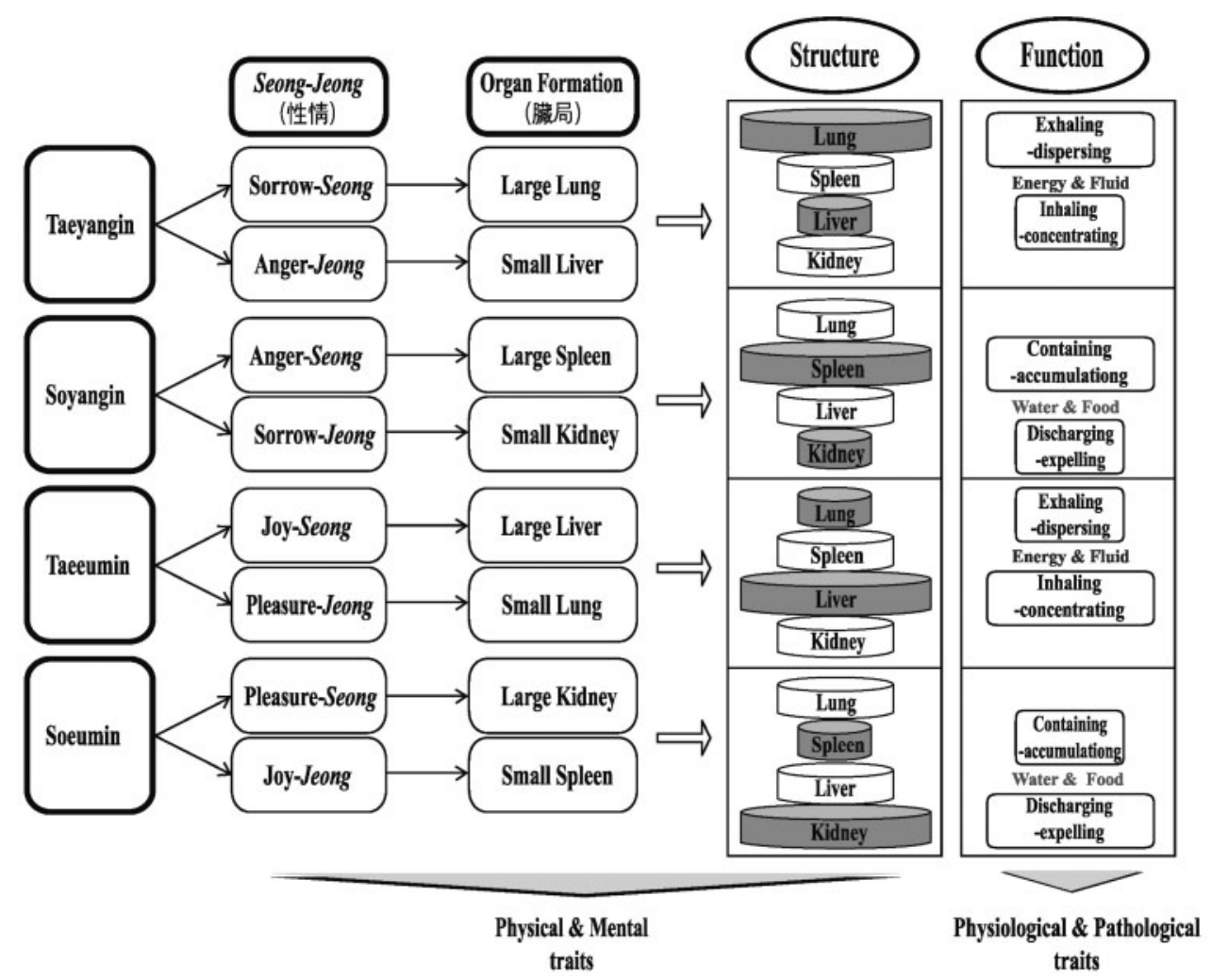

Figure 6. The formation of 'small' and 'large' organ systems and the resultant structural and functional deviation. Each constitutional type has a specific pattern of Seong-Jeong deviation, and this deviation causes the variations in the formation of organ systems in different constitutional types. The constitutionally differentiated organ system scheme gives rise to the constitutionally differentiated physiological traits.

relatively undeveloped. The exterior physical elements associated with the liver system are relatively developed, whereas those associated with the lung system are relatively undeveloped. The inhaling and concentrating of energy and fluid carried out by the liver and small intestine are relatively strong, whereas the exhaling and dispersing of energy and fluid carried out by the lung and esophagus are relatively weak.

The Soyangin and Soeumin share a physiological system based on the relationship and balance between the containing and accumulating function of the spleen system and the discharging and expelling function of the kidney system, two main components of the water-food metabolism. Due to the organ system scheme of a 'large' spleen system and a 'small' kidney system in Soyangin, the structure and function of body components associated with the spleen system are relatively developed, whereas those associated with the kidney system are relatively undeveloped. The exterior physical elements associated with the spleen system are relatively developed, whereas those associated with the kidney system are relatively undeveloped. The containing and accumulating of water and food carried out by the spleen and stomach are relatively strong, whereas the discharging and expelling of water and food carried out by the kidney and large intestine are relatively weak. Due to the organ system scheme of a 'large' kidney system and a 'small' spleen system in Soeumin, the structure and function of body components associated with the kidney system are relatively developed, whereas those associated with the spleen system are relatively undeveloped. The exterior physical elements associated with the kidney system are relatively developed, whereas those associated with the spleen system are relatively undeveloped. The discharging and expelling of water and food carried out by the kidney and large intestine are relatively strong, whereas the containing and accumulating of water and food carried out by the spleen and stomach are relatively weak (Fig. 6).

\section{Requisite Energy（保命之主）}

The requisite energy is the constitutionally differentiated functional energy required to maintain health and preserve life. The requisite energy is related to the 'small' organ in each constitutional type: the inhaling and concentrative energy becomes the requisite energy in the Taeyangin (who has a 'large' lung system and a 'small' liver system) due to the 'small' liver system and the resultant weakness in the inhaling and concentrative functions; the exhaling and dispersive energy becomes the requisite energy in the Taeeumin (who has a 'large' liver 
system and a 'small' lung system) due to the 'small' lung system and the resultant weakness in the exhaling and dispersive functions; the clearing eum (Yin) energy becomes the requisite energy in the Soyangin (who has a 'large' spleen system and a 'small' kidney system) due to the weakness in the discharging and expelling functions and the lack of cold energy that serves as the basic structural and functional unit of the kidney system; the warming Yang energy becomes the requisite energy in the Soeumin (who has a 'large' kidney system and a 'small' spleen system) due to the weakness in the containing and accumulating functions and the lack of warm energy that serves as the basic structural and functional unit of the spleen system. The requisite energy is the basic energy needed by each constitutional type in order to maintain homeostasis. The concept of the requite energy provides the basic rationale that explains the SCM physiology and pathology.

\section{Constitutionally Differentiated Diagnosis and Treatment of Disease}

Contrary to the uniform anatomy and physiology found in Western medicine, the SCM uses a constitutional typology system that types individuals into four different constitutional types. This system of constitutional typing, derived from the theoretical backgrounds explained in this article, describes different structural and functional characteristics of the human body for different constitutional types. This variation manifests in different morphological (7-14), psychological $(15,16)$, physiological (17-20) and pathological (21-24) characteristics. Many studies support this constitutional variation-for example, there have been reports of significant difference in the prevalence of chronic disease across different constitutional types (21-23). Because of this physiological and pathological variation, the same disease is often treated differently for different individuals based on the constitutionally differentiated traits in clinical practice. Though large-scale studies are yet to be carried out, the effectiveness of such constitutionally differentiated treatment has been confirmed in various small-scale studies (25-29).

\section{Discussion}

SCM was forged within the philosophical backgrounds of one of the most deep-rooted ethical teachings in human history, Confucianism and is in a sense a medical expansion of the Korean Neo-Confucianism (30). Although TKM had been influenced by TCM, it managed to develop certain distinctive features of its own over time. In the early 17 th century, the renowned Korean physician Heo Jun compiled a monumental book, Donguibogam, thereby triggering a turning point, wherein TKM veers off from the conventional TCM tradition and begins to construct an independent medical system. One of the main distinctions of TKM was that TKM focused on the characteristics of the afflicted individual rather than on the isolated symptoms. This viewpoint was emphasized and fortified by Lee Je-ma, author of Donguisusebowon, in the early 20 th century (31).

TCM is based on the Yin-Yang theory and the fivephase theory. The organs of the body are discussed always within the context of their relationship with the five elemental phases, with the other organs, and with the other components of the body. The TCM physiology and pathology adheres to the concept of the five Yin organs (five viscera) and the six Yang organs (six bowels) (32).

The SCM classifies individuals into four different constitutional types (TY type, TE type, SY type and SE type) and describes their respective physiology, pathology, therapeutics and health preservation methods (33). This classification of individuals into four constitutional types is based on its unique perspective of the human body that differs from the conventional TCM viewpoint. To propound, SCM summarizes the structure and functions of the human body within the theoretical background of the quadrifocal Sasang scheme, setting it apart from conventional TCM, and bases its explanation of the differentiation into four constitutional types on this rationale. SCM looks beyond the general structural and functional characteristics of the body and emphasizes the individual idiosyncratic characteristics.

This SCM perspective on the structure and function of the human body emphasizes the close structure-function association, and this gives rise to the holistic perception of the human body found in SCM and is used to explain its typical physiology and pathology. There have been recent attempts in modern physiology to combine structure and function and understand the human body on a holistic level, of which the most well-known is the 'Physiome'. Physiome is a new approach in understanding human biology and life that differs from conventional molecular biology methods. Physiome promotes integrative and holistic methods and is becoming progressively more widespread throughout the world (34). Physiome is a term coined by Prof. Bassingthwaighte of the University of Washington, derived from the Latin roots 'physio', meaning life and 'ome', meaning whole, which together translate into 'life as a whole'. Physiome models can be found in SCM in that it attempts to understand the human body on a holistic level (34).

On the basis of its unique view on the structure and function of the human body, SCM has developed and expanded a constitutional typology system that explains the individual physical attributes. Due to the structural and functional variations among the constitutional types, each constitutional type is required to secure a specific 'requisite energy' in order to maintain health and preserve life. This 'requisite energy' can also be translated as the constitutionally differentiated fortifying energy, 
the functionality of which can determine the degree of health and illness in an individual.

The term homeostasis is used by physiologists to indicate the maintenance of equilibrated or constant, conditions in the internal environment of the human body. Essentially, the functions of all the organs and tissues of the body are aimed to maintain these constant conditions (35). The laws of homeostasis and the necessary physiological functions are absolute and universal in all individuals. However, in SCM, the physiological and pathological characteristics differ between individuals, and the conditions for maintaining perfect homeostasis are different in different constitutional types.

The SCM is a traditional Korean medical theory that differs greatly from conventional TCM in its theoretical basis, rationale and especially in its fundamental perspective on the structure and function of the human body. Therefore, understanding the SCM viewpoint of the human body is necessary to properly understand the SCM and fully appreciate its advantages as the individualized medicine that focuses on the particular idiosyncrasies found in different individuals.

\section{Funding}

Korea Science and Engineering Foundation (KOSEF) grant funded by the Korean government (MEST) (Grant No. M10643020001-08N4302-00100).

\section{References}

1. Song IB, Koh BH, Lee EJ, Kim KY, Kim DR, Park SS, et al. Sasang Constitutional Medicine. 2nd edn. Seoul: Jipmoondang, 2004, 325-35 (in Korean).

2. Shim BS, Koh BH, Ahn KS. Education in oriental medicine in Kyung Hee University. eCAM 2004;1:331-4.

3. Yoo JH, Kim JW, Kim KK, Kim JY, Koh BH, Lee EJ. Sasangin diagnosis questionnaire: test of reliability. J Altern Complement Med 2007;13:111-22.

4. Lee JM. Donguisusebowon, 8th edn. Seoul: Daeseong Publishing, 1998 (in Chinese).

5. Lee JM, Choi SH (trans.). Longevity and Life Preservation in Oriental Medicine. Seoul: Kyung Hee University Press, 1996.

6. CHO HS. Modern approach of the discourse on viscera and bowls and retrogressive disorder. J Sasang Constitut Med 2000;12:84-100 (in Korean).

7. Kim JW, Jeon SH, Sul YK, Kim KK, Lee EJ. A study on the body shape classified by Sasang Constitutions and gender using physical measurements. J Sasang Constitut Med 2006;18:56-61 (in Korean).

8. Huh MH, Koh BH, Song IB. The body measuring method to classify Sasang constitutions. J Sasang Constitut Med 2002;14:51-66 (in Korean).

9. Park EK, Park SS. A morphological study of hand and foot according to the Sasang Constitution. J Sasang Constitut Med 1999;11: 195-207 (in Korean).

10. Hong SC, Lee SK, Lee EJ, Han GH, Chou YJ, Choi CS, et al. A study on the morphologic characteristics of each constitution's trunk. J Sasang Constitut Med 1998;10:101-42 (in Korean).

11. Lee EJ, Pyeon YB, Kwak CK, Yoo JH, Kim JW, Kim KK, et al. The study of face model and face type. J Sasang Constitut Med 2006;18:25-33 (in Korean).

12. Lee EJ, Son EH, Yoo JH, Koh BH, Song IB, Kim JW, et al. The study of Sasangin's face. J Sasang Constitut Med 2005;17:55-68 (in Korean).
13. Kim DR. A study about five-sounds (Gong, Sang, jiao, zhi, yu) of Sasang constitutional sound analysis. J Sasang Constitut Med 2003:15:50-9 (in Korean).

14. Yang SM, Kim SH, Yoo JS, Kim HS, Lee YH, Kim DR. A study on the correlation between sound characteristic and Sasang constitution by pitch range and bandwidth. J Sasang Constitut Med 2001;13:31-9 (in Korean).

15. Chae H, Lyoo IK, Lee SJ, Cho S, Bae H, Hong M, et al. An alternative way to individualized medicine: psychological and physical traits of Sasang typology. J Altern Complement Med 2003;9:519-28.

16. Park HG, Lee JH. A study on the associations between Lee Je Ma Sasang constitutions and C.G. Jung's psychological types. J Sasang Constitut Med 1998;10:41-50 (in Korean).

17. Choi SM, Chi SE, Jung BY, Sung HJ, Ahn KS, Koh BH. A study on the association between Sasang constitutions and body composition. J Sasang Constitut Med 2001;13:148-59. 24-34 (in Korean).

18. Chi SE, Han SK, Choi SM. Theoretical study on the quantification of constitutional information using bioinformatics. $J$ Sasang Constitut Med 2001;13:17-23 (in Korean).

19. Han SK, Chi SE, Chi SM. Study on the analysis of constitutional genes by HLA typing. J Sasang Constitut Med 2001;13:97-103 (in Korean).

20. Sung HJ, Chi SM, Chi SE, Kim MH, Kim HS, Lee EJ, et al. Study on Sasang constitutional medicine by the theory of thermometabolism and hematopoietic-immune system. Seoul: Korea Institute of Oriental Medicine, 1999 (in Korean).

21. Lee KS, Seok JH, Kim SH, Kim YH, Lee SK, Lee EJ, et al. A casecontrol study on risk factors of obese patients of each Sasang constitution. J Sasang Constitut Med 2007;19:94-112 (in Korean).

22. Lee TG, Lee SK, Choe BK, Song IB. A study on the prevalences of chronic diseases according to Sasang constitution at a health examination center. J Sasang Constitut Med 2005;17:32-45 (in Korean).

23. Hwang MW, Lee SK, Choe BK, Song IB, Koh BH. The research on the Sasang constitutional characteristics of stroke inpatients. J Sasang Constitut Med 2005;17:103-19.

24. Lee JH, Kim SH, Lee EJ, Song IB, Koh BH. A study on the correlation of metabolic and intima-media thickness of common carotid artery with Sasang constitution. J Sasang Constitut Med 2007;19:148-59 (in Korean).

25. Lee BJ, Lee JH, Kim SB, Park GS, Jeong YJ, Lee EJ, et al. The clinical study on the effect of Sasang constitutional medical therapy for Taeumin patients in acute stroke. J Sasang Constitut Med 2001;13:23-30

26. Yoo JH, Lee EJ, Kwak CK, Sohn EH, Koh BH, Song IB, et al. Clinical trial of herbal formula on weight loss in obese Korean children. Am J Chin Med 2005;33:713-22.

27. Shin HY, Jeong HJ, Lee JH, Joo JC, Kim KY, Song HJ, et al. Regulatory effect of cytokine production in patients with cerebral infarction by Yulda-Hanso-Tang. Immunopharmacol Immunotoxicol 2000;22:183-93.

28. Jeong HJ, Hong SH, Park HJ, Kweon DY, Lee SW, Lee JD, et al, Yangkyuk-Sanhwa-Tang induces changes in serum cytokines and improves outcome in focal stroke patients. Vascul Pharmacol 2002;39:63-8.

29. Jeong HJ, Lee HJ, Hong SH, Kim HM, Um JY. Inhibitory effect of Yangkyuk-Sanhwa-Tang on inflammatory cytokine production in peripheral blood mononuclear cells from the cerebral infarction patients. Int $J$ Neurosci 2007;117:525-37.

30. Yeo I. Ethnics of the body-A study on the ethical meanings of body in Spinoza and Yi Jae-ma. Uisahak 1998;7:179-97 (in Korean).

31. Cha WS, Oh JH, Park HJ, Ahn SW, Hong SY, Kim NI. Historical difference between traditional Korean medicine and traditional Chinese medicine. Neurol Res 2007;29(Suppl 1):S5-9.

32. Ted J Kaptchuk. The Web that has No Weaver - Understanding Chinese Medicine. NewYork: Congdon \& Weed, Inc., 1983, 53.

33. Song IB, Joseph K. Kim, Huh MH (trans). An Introduction to Sasang Constitutional Medicine. Paju, Korea: Jipmoondang, 2005, 97.

34. Shim EB, Lee SW, Kim JY, Earm YE. Physiome and Sasang constitutional medicine. J Physiol Sci 2008;58:433-40.

35. Guyton AC. Textbook of Medical Physiology, 8th edn. Philadelphia, USA: W.B. Saunders Company, 1991.

Received March 14, 2009; accepted June 18, 2009 


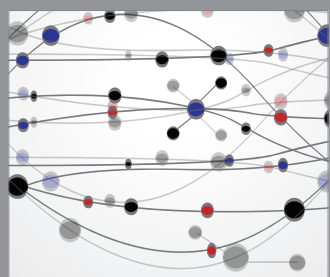

The Scientific World Journal
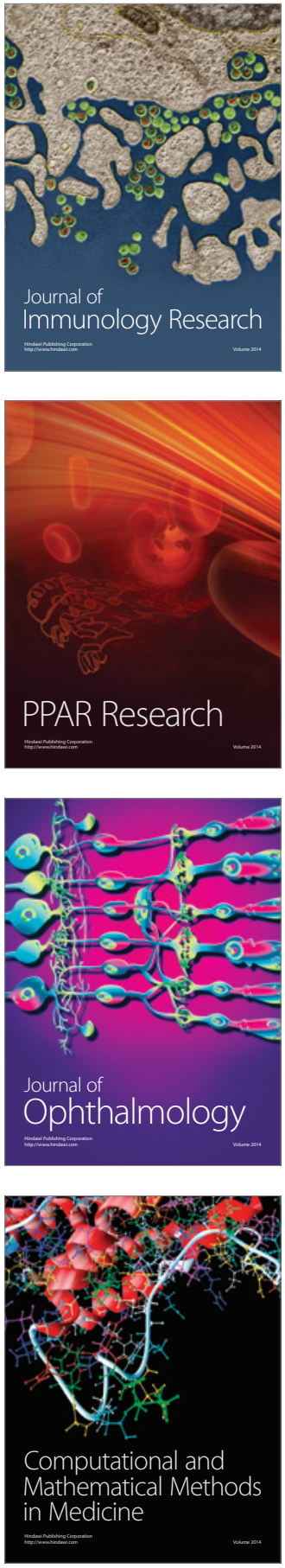

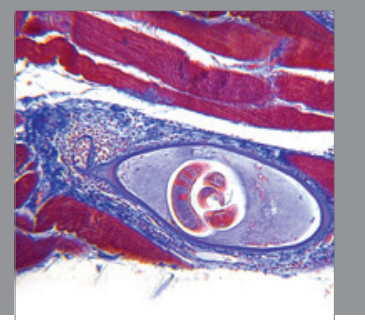

Gastroenterology

Research and Practice
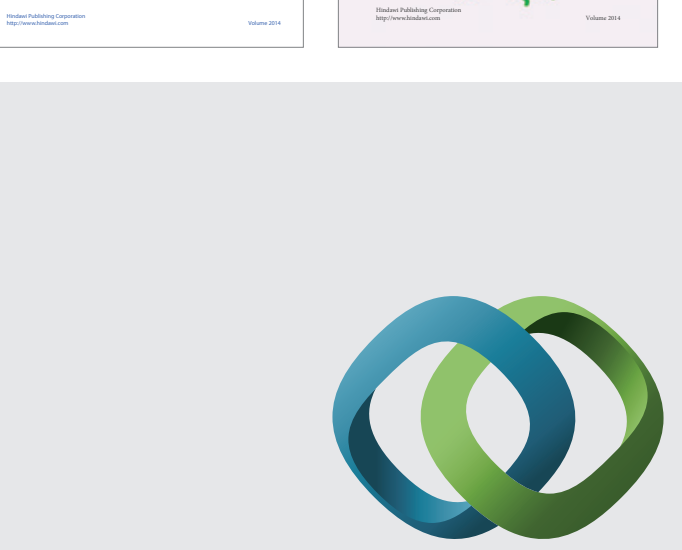

\section{Hindawi}

Submit your manuscripts at

http://www.hindawi.com
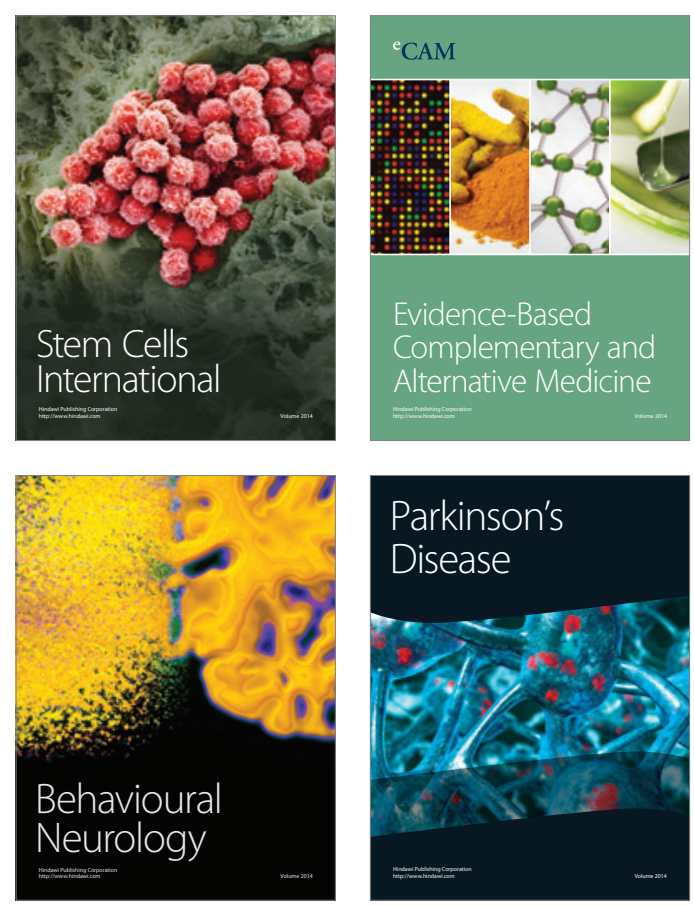

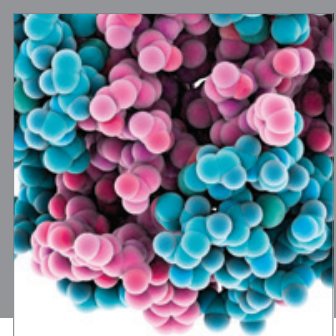

Journal of
Diabetes Research

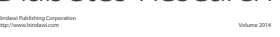

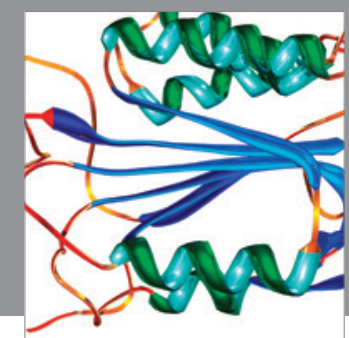

Disease Markers
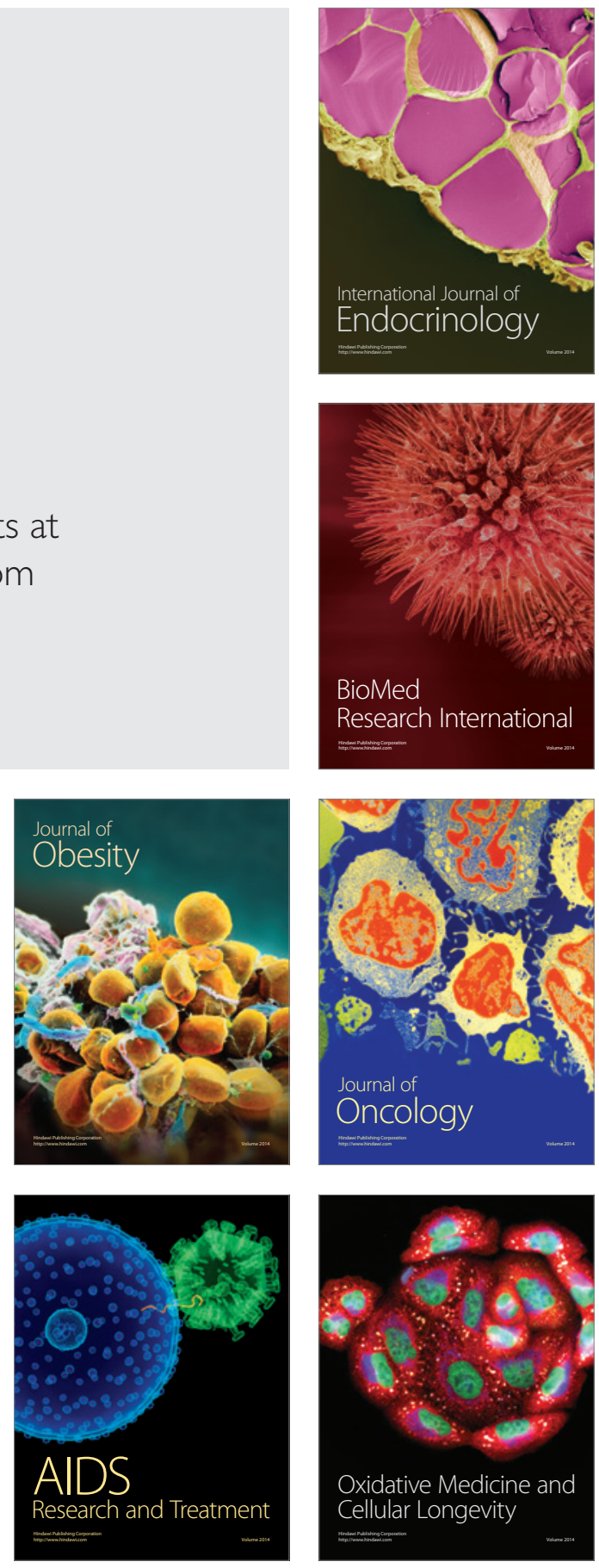